\title{
STRUCTURE THEOREM FOR $A$-COMPACT OPERATORS
}

\author{
G. D. LAKHANI
}

\begin{abstract}
A contraction $T$ defined on a complex Hilbert space is called $A$ compact if there exists a nonzero function $f$ analytic in the open unit disc and continuous on the closed disc such that $f(T)$ is a compact operator. In this paper, the factorization of $f$ is used to obtain a structure theorem which describes the spectrum of $T$.
\end{abstract}

Introduction. A bounded linear operator $T$ on a complex Banach space $X$ is called polynomially compact if there is a nonzero polynomial $p(z)$ such that $p(T)$ is compact. The spectrum of polynomially compact operators $T$ has been completely described by F. Gilfeather [4] by showing that $T$ is a finite sum of power compact operators. A similar problem of describing the spectrum arises in case $p(z)$ is replaced by the uniform limit of polynomials. More explicitly, let $T$ be a contraction defined on a Hilbert space, and $A$ be the uniform closure of polynomials in the supremum norm over $\bar{D} ; D$ is the open unit disc. The elements of $A$ are analytic functions in $D$ which have continuous extension to $\bar{D}$. For $f(z)=\sum a_{n} z^{n} \in A, f(T)=\sum a_{n} T^{n}$ converges in norm, and $\|f(T)\|$ $\leqslant\|f\|_{\infty}$. If $f \in H^{\infty}, f_{r}(z)=f(r z)$ belongs to $A$ for each $r, 0<r<1$, and $\lim _{r \rightarrow 1-0} f_{r}(T)$ exists in the norm. Define $f(T)=\lim _{r \rightarrow 1-0} f_{r}(T)$. For $f \in A$, this definition is consistent. The contraction $T$ is called $A$-compact if there exists a nonzero $f \in A$ such that $f(T)$ is a compact operator. The problem is to describe the spectrum of $A$-compact contractions.

Let $T$ be an $A$-compact contraction on a complex Hilbert space. Denote by $J(T)$ the set of functions $f \in A$ such that $f(T)$ is compact. It is easily seen that $J(T)$ is a nonzero closed ideal of $A$, indeed, it is a principal ideal [5, Corollary, p. 88]. Let $Z$ be the set of common zeros of functions in $J(T)$ in $\bar{D}$. Every function which generates $J(T)$ is of the form $f=F g$ where $F$ is the greatest common divisor of the inner parts of functions in $J(T)$ and $g$ is the outer part of any function which belongs to $A$ and vanishes precisely on $Z \cap\{|z|=1\}$. A generator $f$ of $J(T)$ is called a minimal function of $T$ if its singularities which lie on the unit circle are contained in $Z$. A minimal function $f$ always exists.

An operator $T$ defined on a Hilbert space $X$ is said to be decomposed if there exists a pair $\left(X_{1}, X_{2}\right)$ of subspaces of $X$ such that $X$ has unique decomposition in $\left(X_{1}, X_{2}\right)$ and $X_{i}$ is invariant under $T, i=1,2$. A decomposition of $T$ can be obtained by the decomposition theorem [7, p. 419] if $\sigma(T)$ has more than one

Received by the editors August 29, 1972 and, in revised form, July 15, 1975.

AMS (MOS) subject classifications (1970). Primary 47A65, 47B99.

Key words and phrases. Polynomially compact operators. 
connected component. Let $\lambda$ be an isolated point of $\sigma(T)$, and $\Gamma$ be a rectifiable, simple, closed curve enclosing no point of $\sigma(T)$ other than $\lambda$ and containing no point of $\sigma(T)$. Then

$$
E_{\lambda}=-\frac{1}{2 \pi i} \int_{\xi \in \Gamma}(T-\xi I)^{-1} d \xi
$$

is a projection on $X_{\lambda}=E_{\lambda} X$ along $X_{0}=\left(I-E_{\lambda}\right) X$. The pair $\left(X_{\lambda}, X_{0}\right)$ decomposes $T$ into $T_{\lambda}=T / X_{\lambda}$ and $T_{0}=T / X_{0}$, and the spectra of the two restrictions are $\{\lambda\}$ and $\sigma(T)-\{\lambda\}$, respectively. The terms $X_{\lambda}$ and $T_{\lambda}$ will be used afterwards.

A contraction $T$ defined on a Hilbert space $X$ is called completely nonunitary (c.n.u.) if there exists no nonnull reducing subspace on which $T$ is unitary.

1. We now state the structure theorem.

THEOREM. Let $f$ be a minimal function of a A-compact contraction $T$ defined on a complex Hilbert space $X$, and $Z$ be the set of zeros of $f$ in $\bar{D}$. Then (i) $Z \subseteq \sigma(T)$, (ii) $\sigma(f(T))=f(\sigma(T))$, (iii) $\sigma(T)$ is totally disconnected, (iv) points of $\sigma(T)-Z$ are isolated and are eigenvalues with finite dimensional generalized eigenspaces, and $(\mathrm{v})$ if $\lambda \in Z$ is an isolated point of $\sigma(T)$, then $X_{\lambda}$ is infinite dimensional, $\left(T_{\lambda}-\lambda I\right)$ is power compact in case $f$ is analytic at $\lambda ; g_{\lambda}\left(T_{\lambda}\right)$ is quasinilpotent compact operator in case $\lambda$ is a singularity of $f$ and

$$
g_{\lambda}(z)=(z-\lambda) \exp (\rho(z+\lambda) /(z-\lambda)), \quad \rho>0 .
$$

Proof. (i) By the factorization theorem [5, p. 67], $f$ is uniquely expressible in the form $f(z)=B(z) S(z) F(z)$ where $B(z)$ is a Blaschke product of the form

$$
B(z)=r z^{p} \prod_{i}^{\infty}\left(\frac{\bar{\alpha}_{n}}{\left|\alpha_{n}\right|} \frac{\alpha_{n}-z}{1-\bar{\alpha}_{n} z}\right)^{p_{n}},
$$

where $|r|=1$ and $\Pi\left|\alpha_{n}\right|^{p_{n}}$ converges; $S(z)$ is the singular part of the form

$$
S(z)=\exp \left[-\int_{0}^{2 \pi} \frac{e^{i t}+z}{e^{i t}-z} d \mu(t)\right]
$$

where $\mu(t)$ is a bounded, nondecreasing function on $(0,2 \pi]$ such that $\mu^{\prime}(t)=0$ almost everywhere on $(0,2 \pi]$, and $F(z)$ is the outer function of the form

$$
F(z)=\exp \left[\int_{0}^{2 \pi} \frac{e^{i t}+z}{e^{i t}-z} \log \left|f\left(e^{i t}\right)\right| d t\right] .
$$

If possible, let $\lambda \in Z-\sigma(T)$. Then the distance $(\lambda, \sigma(T))>\delta$ for some $\delta>0$. Denote by $M=\{z \in \bar{D}:|z-\lambda| \leqslant \delta\}$. Let $B_{M}(z)$ be the subproduct of $B(z)$ which vanishes in $M$, and

$$
\begin{aligned}
S_{M}(z) & =1 \quad \text { if } M \subset D, \\
& =\exp \left[-\int_{0}^{2 \pi} \frac{e^{i t}+z}{e^{i t}-z} d \nu(t)\right],
\end{aligned}
$$


where $\nu(t)$ is a nondecreasing function such that $\mu(t)=\nu(t)$ if $e^{i t} \in M$ and $\nu^{\prime}(t)=0$ if $e^{i t} \notin M$. Then the function $g(z)=B_{M}(z) S_{M}(z)$ is analytic and does not vanish in some neighbourhood of $\sigma(T)$. Hence, $g(T)$ possesses bounded inverse which implies that $f(T) g(T)^{-1}$ is compact. It means that $f / g \in J(T)$, i.e., $g$ divides (nontrivially) the inner part of $f$ which contradicts the minimality of $f$. Hence $Z \subset \sigma(T)$.

(ii) It is the well-known spectral mapping theorem, and has been proved by Foiaş and Mlak [3, pp. 239-245] for c.n.u. operators. We extend it for general contractions. Let $T=T_{u} \oplus T_{0}$ be the decomposition of $T$ into unitary and c.n.u. parts, and let the corresponding decomposition of $X$ and $f(T)$ be $X=X_{u} \oplus X_{0}$ and $f(T)=f\left(T_{u}\right) \oplus f\left(T_{0}\right)$, respectively. Then, in order to prove (ii), it is enough that $\sigma\left(f\left(T_{u}\right)\right)=f\left(\sigma\left(T_{u}\right)\right)$ so that

$$
f(\sigma(T))=f\left(\sigma\left(T_{u}\right)\right) \cup f\left(\sigma\left(T_{0}\right)\right)=\sigma\left(f\left(T_{u}\right)\right) \cup \sigma\left(f\left(T_{0}\right)\right)=\sigma(f(T)) .
$$

If $X_{u}$ is finite dimensional, it is trivially seen that $\sigma\left(f\left(T_{u}\right)\right)=f\left(\sigma\left(T_{u}\right)\right)$. Assume that $X_{u}$ is infinite dimensional. It then follows from the spectral representation of unitary operators that $T_{u}$ has an infinite dimensional reducing subspace. Therefore, $f\left(T_{u}\right)$, being the uniform limit of polynomials in $T_{u}$, has an infinite dimensional reducing subspace. The compactness of $f\left(T_{u}\right)$ implies that $f\left(T_{u}\right)$ is null. So $\sigma\left(f\left(T_{u}\right)\right)=\{0\}$.

For each $\lambda \in \sigma(T)$ and $r, 0<r<1, f_{r}(\lambda) \in f_{r}\left(\sigma\left(T_{u}\right)\right)=\sigma\left(f_{r}\left(T_{u}\right)\right)$. The subadditivity of commuting operators implies that

$$
\begin{aligned}
\left|f_{r}(\lambda)\right| & \leqslant \text { spectral radius of } f_{r}\left(T_{u}\right) \\
& \leqslant \text { spectral radius of }\left[f_{r}\left(T_{u}\right)-f\left(T_{u}\right)\right]+\text { spectral radius of } f\left(T_{u}\right) \\
& \leqslant\left\|f_{r}\left(T_{u}\right)-f\left(T_{u}\right)\right\|+0,
\end{aligned}
$$

and since $|f(\lambda)|=\lim _{r \rightarrow 1-0}\left|f_{r}(\lambda)\right|$, it follows that $f(\lambda)=0$ for each $\lambda$ $\in \sigma(T)$, i.e., $f\left(\sigma\left(T_{u}\right)\right)=\{0\}$. Hence $f\left(\sigma\left(T_{u}\right)\right)=\sigma\left(f\left(T_{u}\right)\right)$. This proves (ii).

(iii) First, we show that $\sigma(T)-Z$ contains only isolated points of $\sigma(T)$. Since $f(T)$ is compact, and $\sigma(f(T))=f(\sigma(T))$, the only possible accumulation point of $f(\sigma(T))$ can be zero. The continuity of $f$ in $\bar{D}$ implies that the possible accumulation points of $\sigma(T)$ are zeros of $f$ in $\bar{D}$, i.e., $\sigma(T)-Z$ contains only isolated points.

To prove (iii), it is enough to show that $Z$ is totally disconnected. If possible, let $Z$ have a connected component which has more than one point. Then either $Z$ has a point of accumulation in $D$ or contains an arc of the unit circle. In either case $f \equiv 0$, a contradiction. The earlier case follows from the analyticity of $f$ in $D$ and the later case follows from a well-known result of $\mathbf{F}$. and $\mathbf{M}$. Riesz about bounded analytic functions.

(iv) Let $\lambda \in \sigma(T)-Z$. By (iii), $\lambda$ is an isolated point of $\sigma(T)-Z$. By the definition of minimal function, $f$ can be assumed to be analytic in some neighbourhood of $\lambda$. Let $X_{\lambda}$ and $T_{\lambda}$ be as defined by $(*)$. Since $f(\lambda) \neq 0, \sigma\left(T_{\lambda}\right)$ $=\{\lambda\}$ and $\sigma\left(f\left(T_{\lambda}\right)\right)=f\left(\sigma\left(T_{\lambda}\right)\right)$, it follows that $0 \notin \sigma\left(f\left(T_{\lambda}\right)\right)$, or equivalently, $f\left(T_{\lambda}\right)$ has bounded inverse. Moreover, $f\left(T_{\lambda}\right)$ is compact, therefore, its domain $X_{\lambda}$ must necessarily be finite dimensional, say $n>0$. Since $T_{\lambda}$ has a matrix representation with respect to each basis of $X_{\lambda}$, hence $\left(T_{\lambda}-\lambda I\right)^{n}=0$ and 


$$
X_{\lambda}=\left\{x \in X:(T-\lambda I)^{n} x=0\right\} .
$$

This completes (iv).

(v) Let $\lambda \in Z$ be a given isolated point of $\sigma(T),\left(X_{\lambda}, X_{0}\right)$ be the decomposition of $X$ obtained by $(*)$, and $T=T_{\lambda}+T_{0}$. If $X_{\lambda}$ is finite dimensional, then $T_{\lambda}$ is compact, and therefore for each $g \in A, g(T)$ is compact, if and only if, $g\left(T_{0}\right)$ is compact. In particular, if

$$
g(z)=f(z)(z-\lambda)^{-1} \exp (-\rho(z+\lambda) /(z-\lambda)), \quad \rho>0,
$$

then $g\left(T_{0}\right)$ exists because $\lambda \notin \sigma\left(T_{0}\right)$. Moreover $g\left(T_{0}\right)$ is compact which implies that $g \in J(T)$. This contradicts that $f$ is a minimal function of $T$.

Now, consider the case that $f(z)$ is analytic in some neighbourhood of $\lambda$. The set $J\left(T_{\lambda}\right)=\left\{g \in A: g\left(T_{\lambda}\right)\right.$ is compact $\}$ is a nonzero closed ideal in $A$. An application of (i) and (iv) for $T_{\lambda}$ implies that $\lambda$ is the only common zero of functions in $J\left(T_{\lambda}\right)$ in $\bar{D}$. It means that $J\left(T_{\lambda}\right)$ is a primary ideal in $A$ and is generated by $(z-\lambda)^{k}$ for some positive integer $k$ [5, Corollary, p. 88] which proves that $\left(T_{\lambda}-\lambda I\right)^{k}$ is compact, i.e., $\left(T_{\lambda}-\lambda I\right)$ is power compact.

Next, consider that $\lambda$ is a singularity of $f$. In this case, the ideal $J\left(T_{\lambda}\right)$ is generated by $g_{\lambda}(z)=(z-\lambda) \exp (\rho(z+\lambda) /(z-\lambda)), \rho>0$, which implies that $g_{\lambda}\left(T_{\lambda}\right)$ is compact. The quasinilpotency of $g_{\lambda}\left(T_{\lambda}\right)$ follows from the fact that $\sigma\left(g_{\lambda}\left(T_{\lambda}\right)\right)=g_{\lambda}\left(\sigma\left(T_{\lambda}\right)\right)=g_{\lambda}(\{\lambda\})=\{0\}$. It completes the proof of the theorem.

2. We give below two corollaries and an example of an $A$-compact operator which is not polynomially compact.

Example. Let $S(z)=\exp ((z+1) /(1-z))$ and $f(z)=(z-1) S(z)$. Consider the contraction $T$ on the Hilbert space $H(S)=H^{2} \ominus S H^{2}$ defined by

$$
T: g(z) \rightarrow z g(z)-S(z) \hat{g}(0)
$$

where

$$
\hat{g}(0)=\langle g(z),(S(z)-S(0)) / z\rangle_{H(S)} .
$$

Then it follows from [6, Proposition 3.2, p. 119] that $S(T)=0$ and from [6, Theorem 5.1, p. 126] that $\sigma(T)=\{1\}$. Now it is clear from [4, Theorem 1] that $T$ cannot be polynomially compact. However, $T$ is $A$-compact because $f(T)=0$.

Corollary 1. If $T$ is $A$-compact and $\sigma(T) \subset D$, then $T$ is polynomially compact.

Proof. Follows immediately from (i).

Sz.-Nagy and Foias have denoted by $C_{0}$ the class of those c.n.u. operators $T$ for which there exists a nonzero $u \in H^{\infty}$ such that $u(T)=0$, and have described the spectrum of such operators, viz., [6, Theorem 5.1, p. 126]. We prove this result for the related class $\hat{C}_{0}(A)$ defined as

$$
\hat{C}_{0}(A)=\{T:\|T\| \leqslant 1 \text { and for some nonzero } f \in A,
$$

$f(T)$ is a quasi-nilpotent compact operator $\}.$ 
Corollary 2. If $T \in \hat{C}_{0}(A)$, then $\sigma(T)=Z$, the set of zeros of the minimal function of $T$.

Proof. Let $f$ be a minimal function of $T$ for which $f(T)$ is compact and quasi-nilpotent. By (ii), $f(\sigma(T))=\sigma(f(T))=\{0\}$ which implies that $\sigma(T)$ $\subseteq Z$. Adding to (i), $\sigma(T)=Z$.

\section{REFERENCES}

1. W. B. Arveson and J. Feldman, A note on invariant subspaces, Michigan Math. J. 15 (1968), 61-64. MR 36 \#6969.

2. P. R. Chernoff and J. Feldman, Invariant subspaces for analytically compact operators, Michigan Math. J. 17 (1970), 23-27. MR 41 \#282.

3. C. Foiaş and W. Mlak, The extended spectrum of completely non-unitary contractions and the spectral mapping theorem, Studia Math. 26 (1966), 239-245. MR 34 \#610.

4. F. Gilfeather, The structure and asymptotic behavior of polynomially compact operators, Proc. Amer. Math. Soc. 25 (1970), 127-134. MR 41 \#2440.

5. K. Hoffman, Banach spaces of analytic functions, Prentice-Hall Ser. in Modern Analysis, Prentice-Hall, Englewood Cliffs, N. J., 1962. MR 24 \# A2844.

6. B. Sz.-Nagy and C. Foiaş, Analyse harmonique des opérateurs de l'espace de Hilbert, Masson, Paris; Akad. Kiadó, Budapest, 1967; English rev. transl., North-Holland, Amsterdam; American Elsevier, New York; Akad. Kiadó, Budapest, 1970. MR 37 \#778; 43 \#947.

7. F. Riesz and B. Sz.-Nagy, Leçons d'analyse fonctionnelles, 2nd ed., Akad. Kiadó, Budapest, 1953; English transl., Ungar, New York, 1955. MR 15, 132; 17, 175.

Department of Mathematics, Indian Institute of Technology, Kanpur 208016, India

Current address: Digital Computer Laboratory, University of Illinois, Urbana, Illinois 61801 